Check for updates

1 Institute for Evidence Based Healthcare, Bond University, Australia

2 University College London, London, UK

3 Centre for Epidemic Interventions Research, Norwegian Institute of Public Health, Oslo, Norway

4 Faculty of Health Sciences, Oslo Metropolitan University, Oslo, Norway

Correspondence to: P P Glasziou PauL_Glasziou@bond.edu.au

Cite this as: BMJ 2021;375:n2729 http://dx.doi.org/10.1136/bmj.n2729 Published: 18 November 2021

\section{Public health measures for covid-19}

\author{
Lack of good research is a pandemic tragedy
}

Paul P Glasziou, ${ }^{1}$ Susan Michie, ${ }^{2}$ Atle Fretheim ${ }^{3,4}$

Although the pandemic has seen remarkable trials for vaccines and drug treatments, much less has been done to evaluate the effects of public health and social measures (PHSMs) ${ }^{1}$; also known as non-pharmaceutical interventions (NPIs) or behavioural, environmental, social, and systems interventions (BESSIs). ${ }^{1}$ A linked systematic review of public health measures for covid $-19^{2}$ by Talic and colleagues (doi:10.1136/BMJ-2021-068302) found just one randomised controlled trial-of mask wearing-among 35 eligible studies that could provide estimates on the effectiveness of individual interventions. ${ }^{3}$ The 34 observational studies comprised 14 natural experiments or quasi-experiments and nine cohort, two case-control, and nine cross sectional studies-from Asia ( $n=11)$, the United States $(n=9)$, Europe $(n=7)$, and elsewhere $(n=8)$. Combined, these studies suggested relative reductions in incidence of $25 \%$ (relative risk 0.75 , $95 \%$ confidence interval 0.59 to 0.95 ) for physical distancing, 53\% (0.47, 0.29 to 0.75$)$ for mask wearing, and $53 \%$ (0.47, 0.19 to 1.12) for handwashing. The handwashing finding is somewhat surprising, and at odds with evidence that the predominant route of transmission is through inhalation rather than fomites. ${ }^{4}$

What might explain the $53 \%$ reduction associated with handwashing? It is likely that handwashing is a marker for several protective behaviours such as avoiding crowds, distancing, and mask wearing. So, findings from the observational studies might be better interpreted as the impact of a bundle of correlated protective behaviours for which the individual behaviours are a marker. Similar with handwashing, the effects seen in observational studies of mask wearing will be confounded by other protective behaviours not accounted for during adjustment.

Talic and colleagues' review includes just one randomised controlled trial that evaluated mask wearing, and it was too small for a reliable estimate of effect ( $18 \%$ reduction in incidence for the wearer, $95 \%$ confidence interval $-23 \%$ to $46 \%) .{ }^{5}$ The authors mention, but did not include, a large cluster randomised trial of mask wearing from Bangladesh, currently available as a preprint. ${ }^{6}$ The trial randomised 600 villages (341 830 adults) to surgical masks, cloth masks, or control and found a 9\% (95\% confidence interval o\% to $18 \%$ ) relative reduction in symptomatic SARS-CoV-2 infection (measured by seroprevalence) in villages randomised to the mask interventions. Combined, these two randomised trials suggest that mask wearing is responsible for a statistically significant relative risk reduction of about $10 \%$ in incidence (95\% confidence interval $1 \%$ to $18 \%$ )-important but substantially less than that from observational studies. A third large cluster trial (40 ooo participants) from Guinea-Bissau has completed and should report soon. ${ }^{7}$

Conclusions about other PHSMs are even more difficult to draw: Talic and colleagues say "meta-analysis was not possible for the outcomes of quarantine and isolation, universal lockdowns, and closures of borders, schools, and workplaces.” And perhaps most disappointing, the authors identified no studies of increasing ventilation in indoor settings or socialising outdoors. The paucity of published observational or experimental studies on ventilation is one of the research tragedies of the pandemic. ${ }^{8}$ One report from the US Centers for Disease Control and Prevention suggested that improved ventilation in schools (opening windows and doors) was associated with a $35 \%$ reduction in transmission of SARS-CoV-2. Improved ventilation with additional air cleaning measures such as HEPA filters was associated with a $48 \%$ reduction in transmission. But these findings were based only on survey data, with no assessment of the quality of ventilation or air cleaning. ${ }^{9}$ This vital oversight is surely worthy of more and better research.

What can we take from this new review? It might be reasonable to conclude that a bundle of PHSMs is modestly effective but that individual components cannot be reliability assessed owing to lack of adjustment for confounders or use of randomised or factorial trials. ${ }^{10}$ Face masks seem to have a real but small effect for wearer and source control, although final conclusions should await full reports of the trials from Bangladesh and Guinea-Bissau. However, the quality of the current evidence would be graded-by GRADE criteria ${ }^{11}$-as low or very low, as it consists of mainly observational studies with poor methods (biases in measurement of outcomes, classification of PHSM, and missing data), and high heterogeneity of effect size. More and better research are needed.

The most striking finding of Talic and colleagues' systematic review is that only one completed randomised trial of a PHSM was identified, in contrast with the hundreds for trials completed for drug treatments of covid-19 (see www.bessi-collab.net). Considering the central importance of PHSMs for pandemic control, the uncertainties and controversies around their effects, and the immense research effort being put into vaccine and drug development, this lack of investment in public health measures is puzzling-at just $4 \%$ of global research funding for covid-19. ${ }^{12}$ An ongoing survey by the University of Oslo might shed some light on the key barriers to trials of PHSMs and how best to overcome them-we encourage researchers to take part. ${ }^{13}$ Anecdotally, 
barriers include legal and ethical concerns, funding difficulties, and lack of public and political support.

One positive development is a recent initiative by the World Health Organization, backed by the Norwegian government, to strengthen "the global evidence base to provide actionable and evidence-informed guidance on such measures for decision-makers." ${ }^{14}$ This is only achievable if the global community of public health researchers, practitioners, and decision makers are ready and have the capacity to conduct the much needed trials, and funders are ready to fund them.

We have read and understood the BMJ Group policy on declaration of interest and declare the following interests: All authors are members of the BESSI Collaboration (www.bessi-collab.net).

Provenance and peer review: Commissioned; not externally peer reviewed.

1 World Health Organization. Tracking Public Health and Social Measures. www. who.int/emergencies/diseases/novel-coronavirus-2019/phsm

2 Michie S, West R. Behavioural, environmental, social, and systems interventions against covid-19. BMJ 2020;370:m2982. doi: 10.1136/bmj.m2982. pmid: 32723732

3 Talic S, Shah S, Wild H, etal. Effectiveness of public health measures in reducing the incidence of covid-19, SARS-CoV-2 transmission, and covid-19 mortality: systematic review and meta-analysis. BM/2021;375:e068302.

4 Science Brief. SARS-CoV-2 and Surface (Fomite) Transmission for Indoor Community Environments. CDC April 5, 2021. www.cdc.gov/coronavirus/2019-ncov/more/science-and-research/surface-transmission.html

5 Bundgaard H, Bundgaard JS, Raaschou-Pedersen DET, etal. Effectiveness of Adding a Mask Recommendation to Other Public Health Measures to Prevent SARS-CoV-2 Infection in Danish Mask Wearers: A Randomized Controlled Trial. Ann Intern Med 2021;174:335-43. doi: 10.7326/M20-6817. pmid: 33205991

6 Abaluck J, Kwong L, Styczynski A, et al. Normalizing Community Mask-Wearing: A Cluster Randomized Trial in Bangladesh (April 2021). NBER Working Paper No w28734. https://ss rn.com/abstract $=3838500$

7 Bandim Health Project. Locally Produced Cloth Face Mask and COVID-19 Like Illness Prevention. https://clinicaltrials.gov/ct2/show/NCT04471766

8 Hammond A, Khalid T, Thornton HV, Woodall CA, Hay AD. Should homes and workplaces purchase portable air filters to reduce the transmission of SARS-CoV-2 and other respiratory infections? A systematic review. PLoS One 2021;16:e0251049. doi: 10.1371/journal.pone.0251049. pmid: 33914823

9 Mask Use and Ventilation Improvements to Reduce COVID-19 Incidence in Elementary Schools - Georgia. November 16-December 11, 2020. www.cdc.gov/mmwr/volumes/70/wr/mm7021e1.htm

10 Collins LM, Dziak JJ, Kugler KC, Trail JB. Factorial experiments: efficient tools for evaluation of intervention components. Am J Prev Med 2014;47:498-504. doi: 10.1016/j.amepre.2014.06.021. pmid: 25092122

11 GRADE Handbook. https://gdt.gradepro.org/app/handbook/handbook.html

12 RESIN R\&D Data \& Dashboard To Support The Covid-19 Pandemic Response www.theciru.com/resin-covid19

13 Institute of Health and Society. Survey on Barriers and Facilitators to Research Trials of Non-Pharmaceutical Interventions. https://www.med.uio.no/helsam/english/research/projects/Covid-19-Research-Non-Pharmaceutical-Interventions/survey-covid-19-response/

14 Enria D, Feng Z, Fretheim A, etal. Strengthening the evidence base for decisions on public health and social measures. Bull World Health Organ 2021;99:610-610A doi: 10.2471/BLT.21.287054. pmid: 34475594 